\title{
Investigation of the effect of some organic compounds on the formation of an antifriction film in a dioctyl sebacate medium
}

\author{
Igor V. Kolesnikov, Konstantin S. Lebedinskii, and Mikhail V. Boiko* \\ Rostov State Transport University, 344038 Rostov-on-Don Rostovskogo Strelkovogo Polka \\ Narodnogo Opolcheniya Square 2, Russia
}

\begin{abstract}
The influence of addition of malonic acid, diethylmalonate and acetylacetone to dioctylsebacate on the process of antifriction film formation is studied. It was found that the addition of small amounts of diethylmalonate and acetylacetone contributes to the acceleration of the tribosystem output to the regime. The addition of malonic acid slows down the process of forming an anti-friction film. The results suggest that the coordination iron compounds are involved in the process of antifriction film formation.
\end{abstract}

\section{Introduction}

Synthetic ester-based lubricants can be used in almost all friction units of machines and mechanisms due to the opportunity to control the chemical composition of the lubricant at the production stage. By varying the length of the hydrocarbon chain of the alcohol and acid fragment of the ester molecule, as well as changing the number of functional groups, it is possible to achieve the required characteristics of the lubricant for a particular site of application. The ability to form protective antifriction films on the metal surfaces of rubbing bodies is also one of the undeniable advantages of such lubricants [1-6]. Despite the undoubted relevance of the study of the chemical composition of these films, currently there is no consensus on the mechanism of their formation [1-6].

The aim of this work is to study the effect of organic additives on the formation of antifriction films in the medium of dioctylsebacate.

\section{Materials and methods}

Tribological tests of lubricants were carried out on the end friction machine according to the scheme "three pins on the steel disc". Pins and discs were made from AISI 420S steel, roughness was $\mathrm{Ra}=1,5$. The diameter of each pin was $1 \mathrm{~cm}$, and the distance from the center of the disc to the middle of the friction track was $35 \mathrm{~mm}$. For the repeatability of the tribological tests, the pins previously were repeatedly grinded to the steel surface of the disc

\footnotetext{
*Corresponding author: mikle-1@list.ru
} 
with same roughness. All tests began without running-in of rubbing surfaces, considering that the antifriction film formed during the experiment will smooth out the surface roughness of the disk, thereby reducing the friction coefficient. Among the all friction parameters, we controlled only the friction coefficient. The load on the friction unit was $2 \mathrm{MPa}$, the sliding speed was $0,5 \mathrm{~m} / \mathrm{s}$ for all tests.

The presence of the film on the friction surface was confirmed by a scanning electron microscope Zeiss EVO-18 MA equipped with BSD and SE sensors. The accelerating voltage varied from 5 to $15 \mathrm{kV}$. The film is an insulator, and therefore its presence on the metal is clearly detectable. The steel surfaces were pre-treated with a solvent to remove excess of lubricant.

Objects of research were dioctylsebacate (DOS), diethylmalonate, malonic acid and acetylaceton.

\section{Results and discussion}

We have found that, on average, to stabilize the coefficient of friction in the environment of dioctylsebacate requires 5-6 hours, Fig. 1. A gradual decrease in the coefficient of friction in this case is the result of the formation of a surface film on the friction track, which is easily detected after experiment, Fig. 2. The process of stabilization of the coefficient of friction can be reduced with the introduction of small quantities of diethylmalonate in a lubricating medium. However, the addition of a diethylmalonate additive has an ambiguous effect on the process of the friction coefficient stabilizing, Fig. 1, 3.

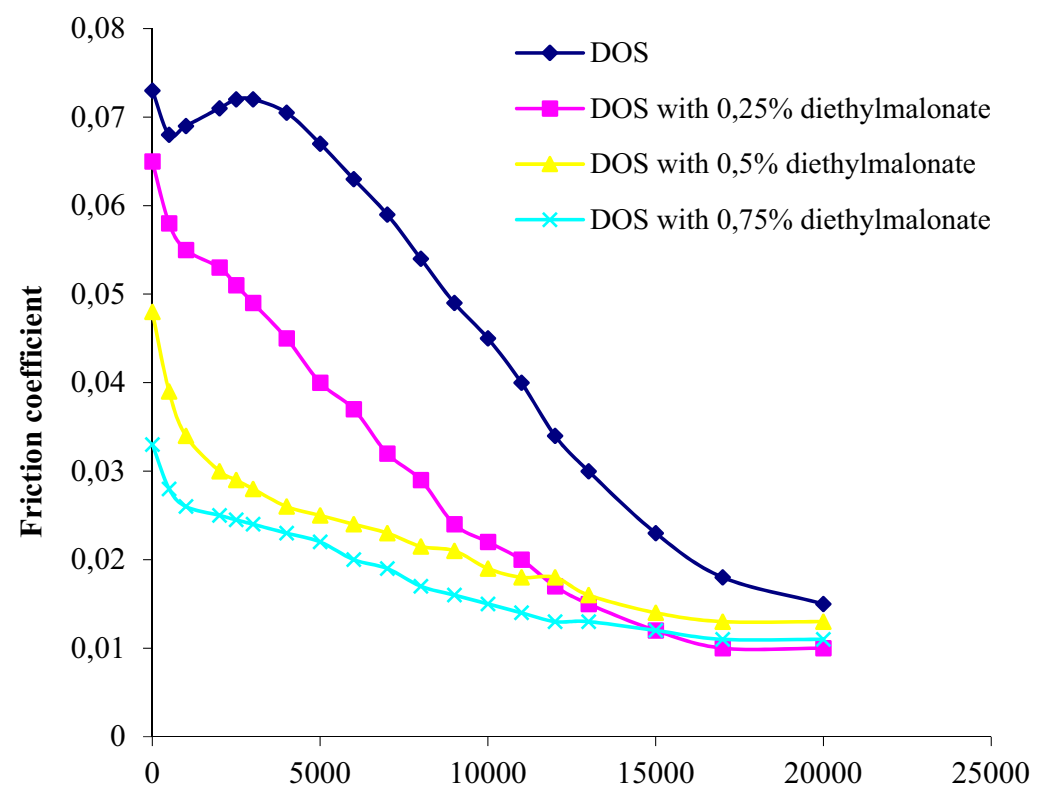

Time, s

Fig. 1. The dependence of the coefficient of friction on time in the presence of pure dioctylsebacate and dioctylsebacate with the addition of diethylmalonate in various concentrations. 


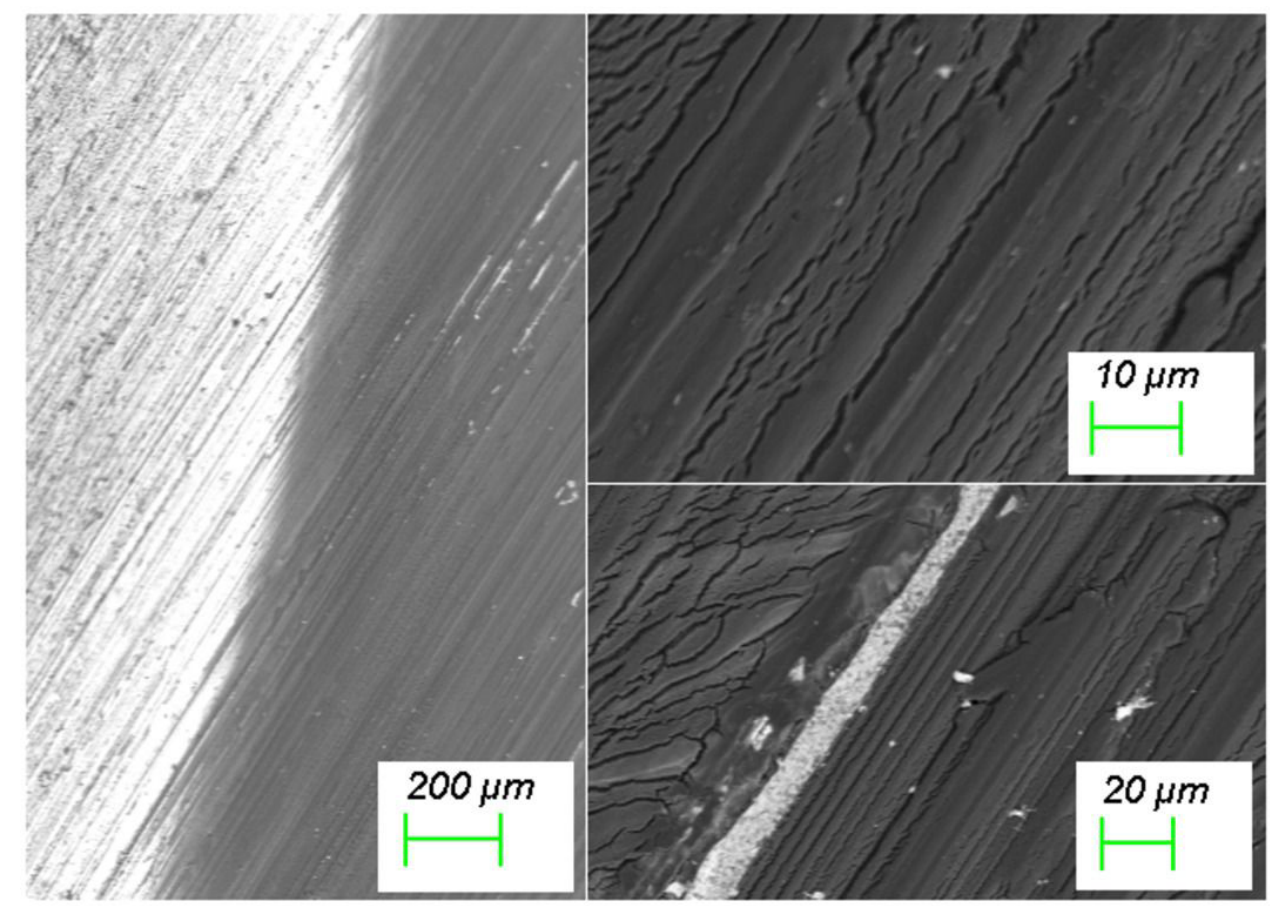

Fig. 2. SEM-images of antifriction film on steel surfaces with different scaling. The dark areas represent the dielectric antifriction film on steel surfaces.

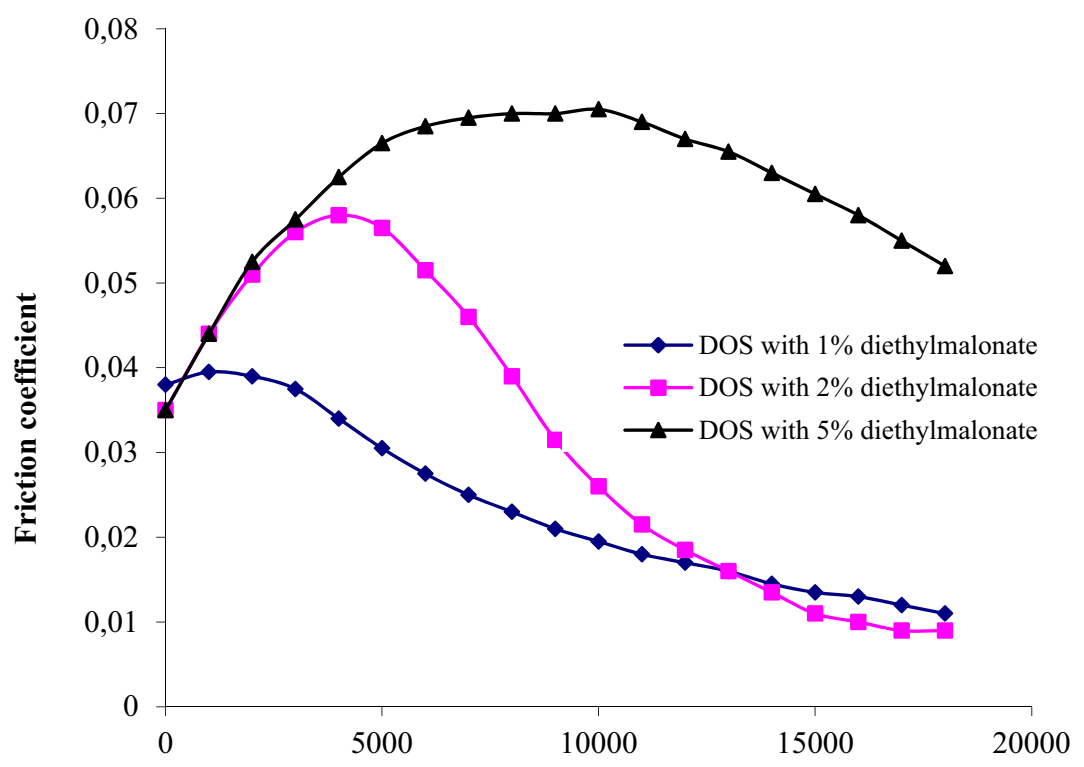

Time, $s$

Fig. 3. The dependence of the coefficient of friction on time in the presence of dioctylsebacate with the addition of diethylmalonate in concentrations exceeding $1 \%$. 
Addition of less than $1 \%$ diethylmalonate significantly decreases the initial coefficient of friction that apparently relates to the interaction of diethylmalonate with the surface of the rubbing bodies. Also, the behavior of the coefficient of friction in the first hour of testing is significantly changed. During this period, the addition of diethylmalonate leads to a sharp decrease in the friction coefficient, while the lubricant without additives is characterized by its increase. These phenomenon lead to the fact that the systems containing diethylmalonate, in general, show a smoother output to a stable regime of friction, however, the final stabilization of the friction coefficient due to a very slow decrease in the last hours of the test takes place only 1-1,5 hours earlier than the system without this ester. To compare the antifriction properties of the additive, we estimated the time for which the friction coefficient in the friction unit reaches a value of 0,02 , that is, when it is more than three times lower than the initial value in the lubricant without additives. This time is about 5000 seconds for $0,75 \%$ ether solution; 9000 seconds - for $0,5 \%$ solution; 11000 seconds - for $0,25 \%$ solution and 16000 seconds-for lubricant without additives.

Fig. 3 shows the same dependencies for systems in which the content of the diethylmalonate exceeds $1 \%$. First of all, it should be noted that the initial points on the charts are the same, which indicates the limit of adsorption saturation of molecules of diethylmalonate of the metal surface. The presence of a sharp rise in the friction coefficient on the graphs, which in the case of a $5 \%$ solution of diethylmalonate leads to a twofold increase in the coefficient of friction compared to the initial, we explain as the special chemical interaction of ester molecules with a metal surface. Diethylmalonate is able to form two coordination bonds with ions of different metals, in particular, with iron [7], which leads to the formation of strong complex compounds. We believe that the presence of a small amount of such complexes in the test environment has a beneficial effect on the formation of antifriction film. However, the formation of a film also needs the interaction of iron ions with the molecules of the DOS. The increase in the friction coefficient with an increase in the concentration of diethylmalonate above $1 \%$ is probably due to the fact that high concentrations of diethylmalonate in the lubricant chemically bind iron, thereby preventing its interaction with the DOS molecules, which leads to a slowdown in the growth of the film.

This assumption is confirmed by the analysis of the composition of used lubricants, collected after each test. The fact is that in the waste lubricant media, we observed the presence of dispersed particles, which make up to $1,5 \%$ of the total weight of the lubricant. The content of particles gradually decreased with increasing concentration of the additive. In the case of a 5\% solution of diethylmalonate in the lubricant after the test, a weak yellowish staining of iron (III) coordination compounds with diethylmalonate was observed instead of mechanical impurities, despite the fact that the temperature in the friction zone due to the high friction coefficient was the highest among all the tests we conducted. This circumstance suggests that the appearance of these particles is not associated with the thermo-oxidative destruction of the lubricant. We believe that they, like the anti-friction film, are formed on rubbing surfaces due to the tribochemical interaction of DOS molecules with metal. At high concentrations of diethylmalonate, due to exorbitant complexation, the interaction of iron with the molecules of the lubricant medium is hindered, which leads not only to a slowdown in the growth of the film, but also, as a consequence, prevents the formation of dispersed particles in the lubricant medium.

Separately, it is necessary to highlight the fact that the final value of the friction coefficient for all studied systems containing diethylmalonate is lower than for pure DOS. This circumstance we explain as that the addition of diethylmalonate slightly changes the mechanism of film formation, and therefore its composition and anti-friction properties. 
On the basis of the studied regularities, we assumed that the formation of the antifriction film is influenced by substances that can form compounds with iron. The addition of $0,1 \%$ acetylacetone in dioctylsebacate, also helps to speed up the stabilization of the tribosystem, Fig. 4. The effect of the addition of $0,01 \%$ malonic acid is the opposite - the process of forming an anti-friction film is significantly stretched in time, Fig. 4. Such a difference we associate with different chemical properties of products of interaction of these substances with iron ions. Diethylmalonate and acetylacetone form coordination compounds with iron, having in their composition activated methylene group $-\mathrm{CH}_{2-}$ [7], and malonic acid - saltlike complexes, which do not contain activated methylene group.

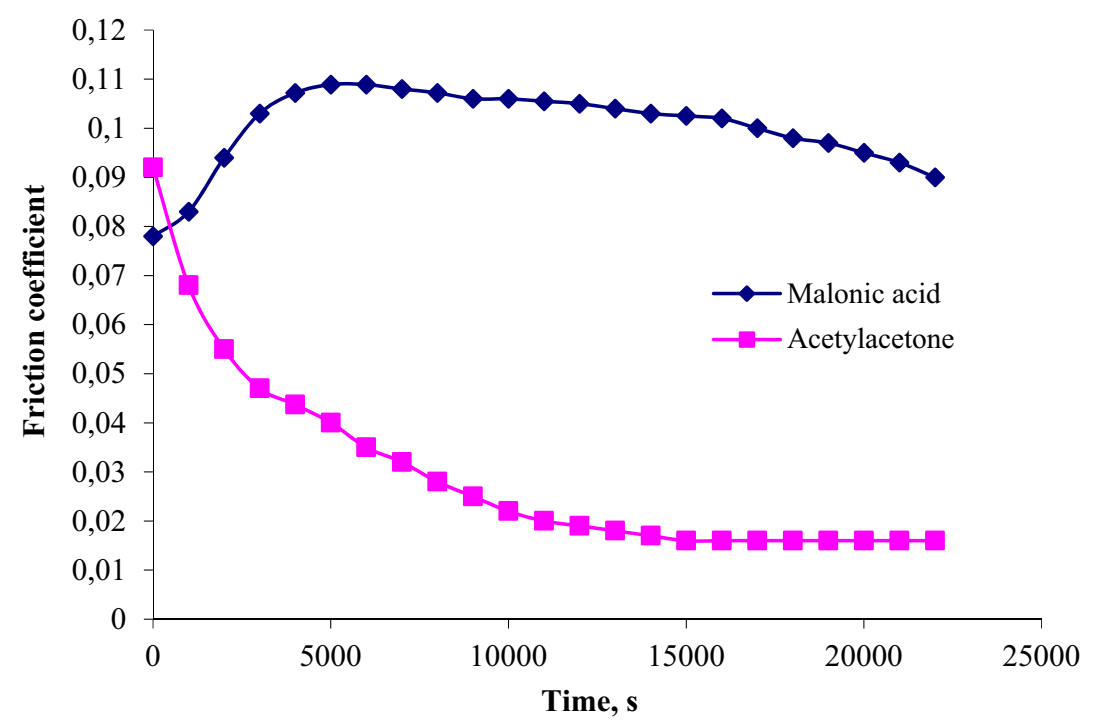

Fig. 4. The dependence of the coefficient of friction on time in the presence of dioctylsebacate with the addition of acetylacetone and malonic acid

\section{Summaries}

It was found that the addition of diethylmalonate to the DOS at low concentrations (up to 1\%) as an additive improves the antifriction properties of the latter by reducing the initial coefficient of friction, accelerating the output of the coefficient to the stable mode, as well as reducing its final value.

The study of the effect of different concentrations of diethylmalonate on the antifriction properties of DOS has found a connection between the nature of mechanical impurities present in the lubricant after the test, and the nature of the anti-friction film on the friction surfaces.

The analysis of the studied regularities allowed stating a hypothesis about the special effect of complex iron compounds on the formation of antifriction films in the DOS environment on rubbing surfaces.

The study has been supported of the Russian Science Foundation (project No. 18-19-00292).

\section{References}

1. S.M, Hsu, R.S. Gates, J. Phys. D: Appl. Phys., 39, 3128 (2006) 
2. E.E. Klaus, E.J. Tewksbury, Lubr. Eng., 29, 11, 205 (1973)

3. S.M. Hsu, R.S. Gates, Tribology International, 38, 305 (2005)

4. R.S. Gates, K.L. Jewett, S.M. Hsu, Tribology Transactions, 32, 4, 423 (1989)

5. A.Y. Grigoriev, I.N. Kavaliova, R. Kreivaitis, A. Kupchinskas, Y. Padgurskas, J. of friction and wear, 37, 6, 552 (2016)

6. A.G. Ponomarenko, G.G. Chigarenko, T.A. Shiryaeva, A.S. Burlov, S.B. Zaichenko, A.D. Garnovskii, V.I. Minkin, A.V. Bicherov, B.G. Konoplev, O.A. Ageev, A.S. Kolomiitsev, V.A. Chetverikova, J. of friction and wear, 31, 5, 387 (2010)

7. J.H. Harwood Industrial Applications of Organometallic Compounds (Chapman and Hall, 1963) 